\title{
Front Matter: Volume 9467
}

, "Front Matter: Volume 9467," Proc. SPIE 9467, Micro- and Nanotechnology Sensors, Systems, and Applications VII, 946701 (24 June 2015); doi: $10.1117 / 12.2202267$

SPIE. Event: SPIE Defense + Security, 2015, Baltimore, MD, United States 


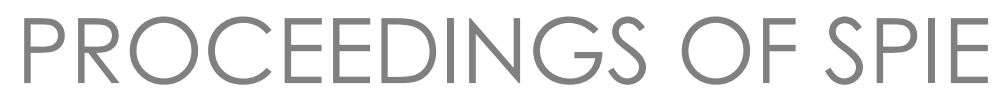

\title{
Micro- and Nanotechnology Sensors, Systems, and Applications VII
}

\author{
Thomas George \\ Achyut K. Dutta \\ M. Saif Islam \\ Editors
}

20-24 April 2015

Baltimore, Maryland, United States

Sponsored and Published by

SPIE 
The papers included in this volume were part of the technical conference cited on the cover and title page. Papers were selected and subject to review by the editors and conference program committee. Some conference presentations may not be available for publication. The papers published in these proceedings reflect the work and thoughts of the authors and are published herein as submitted. The publisher is not responsible for the validity of the information or for any outcomes resulting from reliance thereon.

Please use the following format to cite material from this book:

Author(s), "Title of Paper," in Micro- and Nanotechnology Sensors, Systems, and Applications VII, edited by Thomas George, Achyut K. Dutta, M. Saif Islam, Proceedings of SPIE Vol. 9467 (SPIE, Bellingham, WA, 2015) Article CID Number.

ISSN: 0277-786X

ISBN: 9781628415834

Published by

SPIE

P.O. Box 10, Bellingham, Washington 98227-0010 USA

Telephone +1 3606763290 (Pacific Time) · Fax +1 3606471445

SPIE.org

Copyright (@ 2015, Society of Photo-Optical Instrumentation Engineers.

Copying of material in this book for internal or personal use, or for the internal or personal use of specific clients, beyond the fair use provisions granted by the U.S. Copyright Law is authorized by SPIE subject to payment of copying fees. The Transactional Reporting Service base fee for this volume is $\$ 18.00$ per article (or portion thereof), which should be paid directly to the Copyright Clearance Center (CCC), 222 Rosewood Drive, Danvers, MA 01923. Payment may also be made electronically through CCC Online at copyright.com. Other copying for republication, resale, advertising or promotion, or any form of systematic or multiple reproduction of any material in this book is prohibited except with permission in writing from the publisher. The CCC fee code is 0277-786X/15/\$18.00.

Printed in the United States of America.

Publication of record for individual papers is online in the SPIE Digital Library.

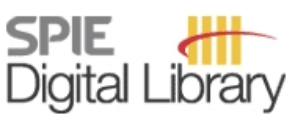

SPIEDigitalLibrary.org

Paper Numbering: Proceedings of SPIE follow an e-First publication model, with papers published first online and then in print. Papers are published as they are submitted and meet publication criteria. A unique citation identifier (CID) number is assigned to each article at the time of the first publication. Utilization of CIDs allows articles to be fully citable as soon as they are published online, and connects the same identifier to all online, print, and electronic versions of the publication. SPIE uses a six-digit CID article numbering system in which:

- The first four digits correspond to the SPIE volume number.

- The last two digits indicate publication order within the volume using a Base 36 numbering

system employing both numerals and letters. These two-number sets start with 00, 01, 02, 03, 04, $05,06,07,08,09,0 A, 0 B \ldots$. OZ, followed by 10-1Z, 20-2Z, etc.

The CID Number appears on each page of the manuscript. The complete citation is used on the first page, and an abbreviated version on subsequent pages. 


\title{
Contents
}

\author{
ix Authors \\ xiii Conference Committee \\ xvii Introduction
}

KEYNOTE SESSION

946702 High power quantum cascade lasers for infrared countermeasures, targeting and illumination, beacons and standoff detection of explosives and CWAs (Keynote Paper) [9467-77]

FLEXIBLE, STRETCHABLE, TRANSIENT ELECTRONICS: WHAT'S NEXT?

946707 Soft electronics for soft robotics (Invited Paper) [9467-5]

946708 Liquid metals as ultra-stretchable, soft, and shape reconfigurable conductors (Invited Paper) [9467-6]

946709 Transformational electronics are now reconfiguring (Invited Paper) [9467-7]

9467 OA Flexible phosphorene devices and circuits (Invited Paper) [9467-9]

9467 OB Fabrication of nanowire electronics on nonconventional substrates by water-assisted transfer printing method (Invited Paper) [9467-10]

9467 OC Stretchable inorganic nanomembrane electronics for healthcare devices (Invited Paper) [9467-11]

\section{LOW-INTENSITY ENERGY DELIVERY FOR BIOMODULATION I}

$9467 \mathrm{OE} \quad 20 \mathrm{kHz}$ ultrasound assisted treatment of chronic wounds with concurrent optic monitoring (Invited Paper) [9467-13]

9467 OF Guiding tissue regeneration with ultrasound in vitro and in vivo (Invited Paper, Best Paper Award 1st Place) [9467-14]

$9467 \mathrm{OH} \quad$ Ultrasound-mediated blood-brain barrier disruption for targeted drug delivery in the central nervous system (Invited Paper) [9467-16]

9467 ol Sustained acoustic medicine: a novel long duration approach to biomodulation utilizing low intensity therapeutic ultrasound (Invited Paper) [9467-17] 
9467 0J Laser phototherapy: the future of medicine (Invited Paper) [9467-120]

9467 OK Antimicrobial blue light therapy for Candida albicans burn infection in mice (Invited Paper) [9467-18]

9467 OM Red/near-infrared light-emitting diode therapy for traumatic brain injury (Invited Paper) [9467-20]

BEYOND GRAPHENE LAYERED MATERIALS AND DEVICES

9467 ON Van der Waals solids: properties and device applications (Keynote Paper) [9467-21]

946700 Synthesis of two-dimensional materials for beyond graphene devices (Invited Paper) [9467-22]

\section{GRAPHENE AND 2D ELECTRONICS AND OPTOELECTRONICS}

9467 OS 2D electronic materials for army applications (Keynote Paper) [9467-26]

9467 OT Two-dimensional materials for low power and high frequency devices (Invited Paper) [9467-27]

9467 OU Increasing the lego of 2D electronics materials: silicene and germanene, graphene's new synthetic cousins (Invited Paper) [9467-28]

9467 OW Graphene and beyond: two-dimensional materials for transistor applications (Invited Paper) [9467-30]

\section{SURFACE ENHANCED SPECTROSCOPIES FOR ULTRASENSITIVE SENSING}

946710 Super-resolution imaging of surface-enhanced Raman scattering hot spots under electrochemical control (Invited Paper) [9467-33]

NOVEL NANOPHOTONIC DEVICES, SENSORS, AND CONCEPTS BASED ON 2D MATERIALS

946713 2D materials and heterostructures for applications in optoelectronics (Invited Paper) [9467-38] 
ORIGAMI: WHERE ART, DEVICES, AND STRUCTURES MERGE

9467 1B PopupCAD: a tool for automated design, fabrication, and analysis of laminate devices (Invited Paper) [9467-46]

9467 1C Hanaflex: a large solar array for space applications (Invited Paper) [9467-47]

MICRO- AND NANO-SENSORS AND MATERIALS FOR OIL AND GAS APPLICATIONS

9467 ID Oil industry first field trial of inter-well reservoir nanoagent tracers (Keynote Paper) [9467-48]

9467 IF Autonomous micro and nano sensors for upstream oil and gas (Invited Paper) [9467-50]

NOVEL HARSH ENVIRONMENT SENSORS FOR ENERGY APPLICATIONS: JOINT SESSION WITH CONFERENCES 9467 AND 9491

$946711 \quad$ Ultra-high temperature fiber optical chemical sensors based on nano-porous metal oxides (Invited Paper) [9467-54]

9467 iJ Phosphor-based fiber optic temperature sensors for harsh environments (Invited Paper) [9467-55]

9467 IK Development of laser-induced breakdown spectroscopy sensor to assess groundwater quality impacts resulting from geologic carbon sequestration (Invited Paper) [9467-56]

9467 IL Temperature dependent behavior of optical loss from hydrogen species in optical fibers (Invited Paper) [9467-57]

$94671 \mathrm{M}$ High temperature, harsh environment sensors for advanced power generation systems (Keynote Paper) [9467-53]

MAST: BIO-INSPIRED CONTROL: JOINT SESSION WITH CONFERENCES 9467, 9468, 9479

946710 Bio-inspired sensing and control for disturbance rejection and stabilization (Invited Paper) [9467-60]

9467 IP Towards high-speed autonomous navigation of unknown environments (Invited Paper) [9467-61]

$94671 Q \quad$ An analysis of small target feature detection limits using optic flow (Invited Paper) [9467-62]

MAST: SCALE LEGGED LOCOMOTION: JOINT SESSION WITH CONFERENCES 9467, 9468, 9479

9467 is Dynamic legged locomotion for palm-size robots (Invited Paper) [9467-63]

$94671 \mathrm{~T} \quad$ Metastable legged locomotion: methods to quantify and optimize reliability (Invited Paper) [9467-64] 
$94671 \mathrm{U}$ Anticipatory control using substrate manipulation enables trajectory control of legged locomotion on heterogeneous granular media (Invited Paper) [9467-65]

9467 IV Data driven models of legged locomotion (Invited Paper) [9467-66]

\section{NOVEL BEAM CONTROL APPLICATIONS AND TECHNIQUES}

$94671 \mathrm{X}$ Ultraspectral imaging and the snapshot advantage (Invited Paper) [9467-68]

9467 IY Design and characterization of a tunable opto-mechatronic system to mimic the focusing and the regulation of illumination in the formation of images made by the human eye (Invited Paper) [9467-69]

946712 Selective disturbance rejection algorithms for mitigating non-common path errors within beam control systems (Invited Paper) [9467-70]

946720 Beam optimization for imaging lidar (Invited Paper) [9467-71]

\section{PHOTONICS RESEARCH AT SPAWAR}

946721 Photonic RF-IF wideband down conversion using optical injection locking (Invited Paper) [9467-72]

946722 Wideband RF channelizer based on parametric combs (Invited Paper) [9467-73]

946723 OCCIMA: Optical Channel Characterization in Maritime Atmospheres (Invited Paper) [9467-74]

946724 Wavelength optimization via retroreflection for underwater free-space optical communication (Invited Paper) [9467-75]

946725 Preparation of novel HTS films and tunnel junctions for advanced C3I sensor applications (Invited Paper) [9467-76]

\section{ULTRA-FAST BANDGAP PHOTONICS}

946726 Detector response to high repetition rate ultra-short laser pulses. I (Invited Paper) [9467-78]

946728 Ultrafast fiber lasers: practical applications (Invited Paper) [9467-80]

946729 Plasmonic nanoantennas for enhanced midwave and longwave infrared imaging (Invited Paper) [9467-81]

\section{THZ PHOTONICS}

9467 2A Terahertz electronics for sensing and imaging applications (Invited Paper) [9467-82] 
9467 2B A compact THz imaging set-up at 750 microns (Invited Paper) [9467-83]

9467 2C Collection efficiency for millimeter and submillimeter wave antenna-coupled detection (Invited Paper) [9467-84]

9467 2D CMOS mm-wave system-on-chip for sensing and communication (Invited Paper) [9467-85]

$94672 \mathrm{E} \quad$ Applications and challenges for MMW and THz sensors (Invited Paper) [9467-86]

$94672 \mathrm{~F} \quad$ Video rate imaging at $1.5 \mathrm{THz}$ via frequency upconversion to the near-IR (Invited Paper) [9467-87]

9467 2G High power MWIR quantum cascade lasers and their use in intra-cavity THz room temperature generation (Invited Paper) [9467-102]

MID-IR LASER PHOTONICS

$946721 \quad$ Approaches to generation of tunable mid-IR ultrafast pulses with fiber sources (Invited Paper) [9467-89]

$94672 \mathrm{~J} \quad$ Long wavelength mid-infrared from mixing two colors from a fiber amplifier (Invited Paper) [9467-90]

9467 2K Mid-IR lasers based on transition metal and rare-earth ion doped crystals (Invited Paper) [9467-92]

LASER CHEMICAL DETECTION: JOINT SESSION WITH CONFERENCES $9467,9455,9486$

$94672 \mathrm{~N} \quad$ Broadband mid-IR frequency comb source for standoff chemical detection (Invited Paper) [9467-94]

946720 Standoff trace detection of explosives with infrared hyperspectral imagery (Invited Paper) [9467-95]

9467 2P Ozone alteration for background references using QCL-based mid infrared standoff spectroscopy (Invited Paper) [9467-96]

$94672 \mathrm{Q}$ Toward the realization of a compact chemical sensor platform using quantum cascade lasers (Invited Paper) [9467-97]

$94672 R \quad$ Detection of trace explosives on relevant substrates using a mobile platform for photothermal infrared imaging spectroscopy (PT-IRIS) (Invited Paper) [9467-98]

$94672 S \quad$ Standoff detection of chemical and biological threats using miniature widely tunable QCLs (Invited Paper) [9467-99] 
$94672 \mathrm{U}$ Lowering contact resistance of graphene FETs with capacitive extension of ohmic contacts for enhanced RF performance [9467-103]

$94672 \mathrm{~W}$ Comb structure analysis of the capacitive sensitive element in MEMS-accelerometer [9467-105]

$94672 X \quad$ A bimorph electrothermal actuator for micromirror devices [9467-106]

$94672 Y$ A novel class of MEMS accelerometers for guidance and control of gun-fired munitions [9467-108]

946730 Thermal conductivity characterization of in-situ fabricated polysilicon nanowires for uncooled thermoelectric infrared detectors [9467-110]

946731 Two-photon absorption based optical logic [9467-1 11 ] 


\title{
Authors
}

Numbers in the index correspond to the last two digits of the six-digit citation identifier (CID) article numbering system used in Proceedings of SPIE. The first four digits reflect the volume number. Base 36 numbering is employed for the last two digits and indicates the order of articles within the volume. Numbers start with 00, 01, 02, 03, 04, 05, 06, 07, 08, 09, OA, OB...0Z, followed by 10-1Z, 20-2Z, etc.

\author{
Abdolvand, Reza, 30 \\ Adleman, James R., 21, 22 \\ Ahn, Edwin S., 1 Z \\ Aidam, R., 20 \\ Akhter, Nahida, 30 \\ Akinwande, Deji, OA \\ Al-Amin, Chowdhury, 2U \\ Angot, Thierry, OU \\ Aragon, Michael, 30 \\ Arevalo, A., 09 \\ Arvanitis, Costas D., $\mathrm{OH}$ \\ Ascencio, Ana, 23 \\ Aukes, Daniel M., 1B \\ Baker, Terrance L., OJ \\ Bawiec, Christopher R., OE \\ Beechem, Thomas E., 29 \\ Belkin, Mikhail A., $2 G$ \\ Berezueva, Svetlana, 2W \\ Bergeron, Alain, 2B \\ Berggren, Susan A. E., 25 \\ Bersch, Brian M., OT \\ Bian, Siyuan, 2J \\ Bogdanova, Yelena, OM \\ Bonnell, Elizabeth, $1 \mathrm{~L}$ \\ Britkov, Igor M., 2X \\ Brodschelm, Andreas, 28, 21 \\ Bronner, W., 20 \\ Buric, Michael, 1I, 1M \\ Byers, Jeff, 2R \\ Byl, Katie, $1 T$ \\ Carreras, Richard A., $1 \mathrm{Z}$ \\ Carson, Cantwell G., $1 \mathrm{~K}$ \\ Cauble, Galen, 23 \\ Chae, Inseok, 2P \\ Chapman, David, IF \\ Chen, Kevin P., 11 \\ Child, Sally Z., OF \\ Comeau, Eric S., OF \\ Conroy, Joseph, $1 Q$ \\ Credle, S., $1 M$ \\ Cruz-Félix, A., IY \\ Dai, Tianhong, OK \\ Dalecki, Diane, OF \\ Davids, Paul S., 29 \\ deGrassie, John, 23 \\ Deutsch, Erik R., $2 S$ \\ Diaz, David, OE \\ Dickey, Michael D., 08 \\ Djeu, Nicholas, $1 \mathrm{~J}$
}

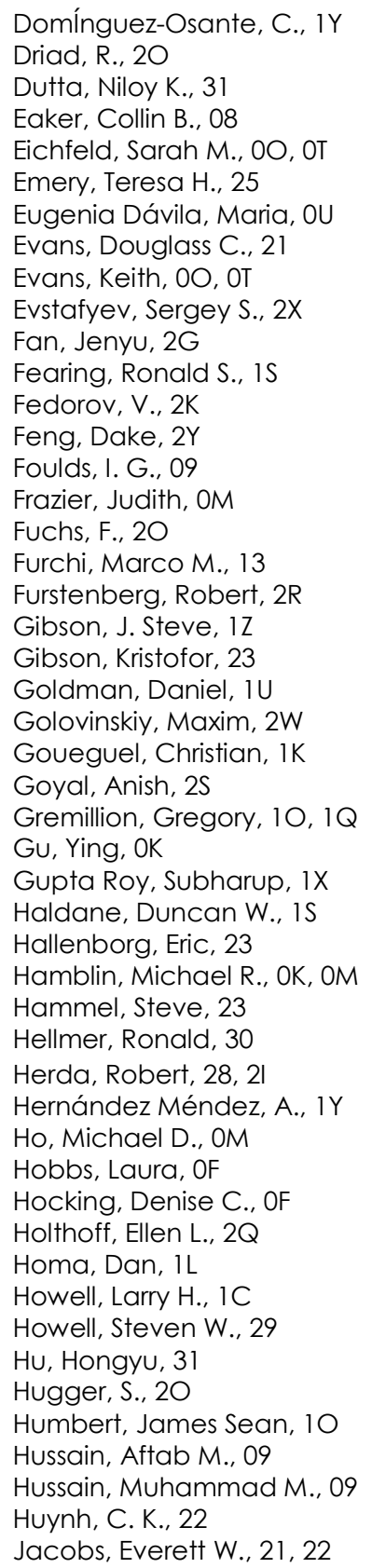


Jain, Jinesh, $1 \mathrm{~K}$

Jarvis, J.-P., 20

Jeon, Inho, 25

Jester, Shai B., 21, 22

Jiang, Aiting, $2 \mathrm{G}$

Jiang, Yifan, $2 G$

Jung, Seungyong, 2G

Kanj, Mazen Y., 1D

Karabiyik, Mustafa, $2 U$

Kashyap, Raman, 2J

Kaul, Anupama B., ON

Kendziora, Christopher A., 2R

Kim, Dae-Hyeong, OC

Kim, Dong Rip, OB

Kim, Jaemin, OC

Kim, Jin K., 29

Knight, Jeffrey A., OM

Koo, Bang-Bon, $0 M$

Korobova, Natalia, 2W

Kosolapov, Andrey, 2W

Kosynkin, Dmitry V., 1D

Kotidis, Petros, $2 S$

Kozlov, Vladimir G., $2 F$

Kramer, Rebecca K., 07

Krengel, Maxine H., OM

Kudenov, Michael W., 1X

Kvalheim, Matthew, $1 \mathrm{~V}$

Lail, Brian A., 2C

Lang, Robert J., 1C

Langer, Matthew D., Ol

Le Lay, Guy, OU

Leach, Jacob H., 0O, 0T

Lee, Chi Hwan, OB

Lee, Yun-Shik, $2 \mathrm{~F}$

Leese de Escobar, Anna M., 25

Leonhardt, Darin, 29

Lewin, Peter A., $\mathrm{OE}$

Lewis, George K. Jr., Ol

Lewis, Robie, $1 \mathrm{M}$

Li, Wenbo, 31

Lin, Chunyan L., 21, 22

Lin, Yu-Chuan, 0O, OT

Livingstone, Margaret, $\mathrm{OH}$

Loranger, Sébastien, 2J

Lyakh, Arkadiy, 02

Magleby, Spencer P., 1C

Maione, Bryan, $1 \mathrm{X}$

Maple, M. Brian, 25

Marchese, Linda E., 2B

Marcus, Logan S., $2 Q$

Margolis, David J., OE

Martin, Paula I., OM

Martyshkin, D., 2K

Mathis, Allison, 1Q

McBryde, Kevin, 23

McDannold, Nathan, $\mathrm{OH}$

McGill, R. Andrew, 2R

Mclntyre, Dustin, $1 \mathrm{~K}$

McNee, lan, 2F

Metzger, Robert, 00, ОT
Mirov, M., 2K

Mirov, S., 2K

Modarres-Zadeh, Mohammad J., 30

Montoya, John A., 29

Moskalev, I., 2K

Mueller, Thomas, 13

Murray, Clinton K., OK

Myslivets, E., 22

Nadkarni, Sumati, OE

Naeser, Margaret A., OM

Nassar, Joanna M., 09

Neidraver, Michael, OE

Neuner, Burton, III, 24

Nguyen, Viet, 2R

Nothwang, William, $1 Q$

Ohodnicki, Paul R., Jr., 1I, 1M

Ohta, Taisuke, 29

O'Regan, Terrance, OS

Ostendorf, R., 20

Pala, Nezih, $2 \mathrm{U}$

Pantalone, Brett, $1 \mathrm{X}$

Papantonakis, Michael, 2R

Pascoguin, B. Melvin, 21, 24

Pastirk, Igor, 28, 21

Patel, C. Kumar N., 02

Pellegrino, Paul M., 2Q

Perconti, Philip, OS

Pereira, Carlos M., $2 Y$

Pérez-Maldonado, Y., $1 Y$

Peters, David W., 29

Pickrell, Gary, 1L

Poole, Zsolt L, 11

Pospischil, Andreas, 13

Power, Chanikarn, $\mathrm{OH}$

Puppe, Thomas, 21

Qian, Feifei, $1 \mathrm{U}$

Radic, S., 22

Raeman, Carol H., OF

Rafailov, Michael K., 26

Rastegar, Jahangir, $2 Y$

Reinhardt, Colin, 23

Revzen, Shai, IV

Richter, Charles, IP

Robinson, Joshua A., 0O, OT

Rojas, Jhonathan P., 09

Roy, Nicholas, IP

Rudiger, Joshua, 23

Ruppert, Lyle, 20

Saglam, Cenk Oguz, IT

Salomon, Eric, OU

Samoilykov, Vyacheslav K., 2X

Sanders-Reed, John N., 2E

Santiago-Alvarado, A., $1 Y$

Schilling, Ch., 20

Schwierz, F., OW

Seachman, S., $1 M$

Sell, Alexander, 28, 2l

Shalimov, Andrew, 2W

Shimoji, Yutaka, 1J

Shur, Michael, 2A 
Sinha, Raju, $2 \mathrm{U}$

Son, Donghee, $0 \mathrm{C}$

Strickland, Donna, 2J

Sunny, Youhan, OE

Tang, Adrian, 2D

Taylor, Benjamin J., 25

Tekavec, Patrick F., 2F

Tereshhenko, Anatolij M., 2X

Terroux, Marc, 2B

Thomson, Mark W., 1C

Thundat, Thomas, 2P

Timoshenkov, Alexey, 2W

Timoshenkov, Sergey P., 2W, 2X

Torres Sevilla, Galo A., 09

Trease, Brian P., 1C

Troccoli, Mariano, 2G

Trybula, Walt, if

Tsintikidis, Dimitri, 23

Van Neste, C. W., 2P

Vasilyev, S., 2K

Vijayraghavan, Karun, 2G

Vodopyanov, Konstantin L., 2F, 2N

Vykhodtseva, Natalia, $\mathrm{OH}$

Wagner, J., 20

Wang, Anbo, $1 \mathrm{~L}$

Wang, Xiaojun, 2G

Wang, Yucheng, OK

Wayne, David, 23

Weber, Maggie L, 10

Weingarten, Michael S., OE

Wendt, Joel R., 29

Wiberg, A. O. J., 22

Willets, Katherine A., 10

Wood, Robert J., 1B

$\mathrm{Xu}$, Yuancheng, 2C

Yan, Aidong, 11

Yang, Q. K., 20

Yee, Megan K., OM

Yogeesh, Maruthi N., OA

Yu, Li, IL

Zach, Armin, 28, 21

Zafonte, Ross, OM

Zaghloul, Mohamed, 11

Zakharova, I. K., 26

Zarrouk, David, $1 \mathrm{~s}$

Zaum, F., 20

Zhang, Kehao, 00, OT

Zhang, Xiang, 31

Zhang, Yongzhi, $\mathrm{OH}$

Zhang, Yunsong, OK

Zheng, Xiaolin, $\mathrm{OB}$

Zhu, Weinan, OA

Zirbel, Shannon A., 1C

Zlatanovic, S., 22

Zubkov, Leonid, OE

Zuev, Egor, 2W

Proc. of SPIE Vol. 9467 946701-11 
Proc. of SPIE Vol. $9467946701-12$

Downloaded From: https://www.spiedigitallibrary.org/conference-proceedings-of-spie on 26 Apr 2023 Terms of Use: https://www.spiedigitallibrary.org/terms-of-use 


\section{Conference Committee}

Symposium Chair

Nils R. Sandell Jr., Strategic Technology Office, DARPA (United States)

Symposium Co-chair

David A. Logan, BAE Systems (United States)

Conference Chairs

Thomas George, ChromoLogic, LLC (United States)

Achyut K. Dutta, Banpil Photonics, Inc. (United States)

M. Saif Islam, University of California, Davis (United States)

Conference Program Committee

Roger Appleby, Queen's University Belfast (United Kingdom)

Debjyoti Banerjee, Texas A\&M University (United States)

Michael P. Buric, National Energy Technology Laboratory

(United States)

Richard Conroy, National Institutes of Health (United States)

Ertugrul Cubukcu, University of Pennsylvania (United States)

Aykutlu Dana, Bilkent University (Turkey)

Nibir K. Dhar, U.S. Army Night Vision \& Electronic Sensors Directorate (United States)

Muhammad M. Hussain, King Abdullah University of Science and Technology (Saudi Arabia)

Matthew E. L. Jungwirth, Honeywell Defense and Space Electronic Systems (United States)

Anupama B. Kaul, National Science Foundation (United States)

Christopher M. Kroninger, U.S. Army Research Laboratory (United States)

Susan M. Maley, U.S. Department of Energy (United States)

Michael C. McAlpine, Princeton University (United States)

Parvaneh Mokarian-Tabari, University College Cork (Ireland)

William D. Nothwang, U.S. Army Research Laboratory (United States)

Stergios J. Papadakis, Johns Hopkins University Applied Physics Laboratory, LLC (United States)

Michael K. Rafailov, The Reger Group (United States)

Bilge Saruhan-Brings, Deutsches Zentrum für Luft- und Raumfahrt (Germany)

Antonio Sastre, National Institutes of Health (United States) 
Noriko Satake, University of California, Davis Medical Center (United States)

Sivalingam Sivananthan, University of Illinois at Chicago

(United States)

Andre U. Sokolnikov, Visual Solutions and Applications (United States)

Kyung-Ah Son, HRL Laboratories, LLC (United States)

Thomas G. Thundat, University of Alberta (Canada)

Richard Vaia, Air Force Research Laboratory (United States)

Christopher C. Wilcox, U.S. Naval Research Laboratory (United States)

Joyce Wong, Schlumberger Ltd. (United States) and California Institute of Technology (United States)

Eui-Hyeok Yang, Stevens Institute of Technology (United States)

Session Chairs

1 Flexible, Stretchable, Transient Electronics: What's Next?

Muhammad M. Hussain, King Abdullah University of Science and Technology (Saudi Arabia)

2 Low-Intensity Energy Delivery for Biomodulation I

Richard Conroy, National Institutes of Health (United States)

3 Low-Intensity Energy Delivery for Biomodulation II

Richard Conroy, National Institutes of Health (United States)

4 Beyond Graphene Layered Materials and Devices

Thomas George, ChromoLogic, LLC (United States)

5 Graphene and 2D Electronics and Optoelectronics

Kyung-Ah Son, HRL Laboratories, LLC (United States)

6 Surface Enhanced Spectroscopies for Ultrasensitive Sensing

Aykutlu Dana, Bilkent University (Turkey)

7 Novel Nanophotonic Devices, Sensors, and Concepts Based on 2D Materials

Ertugrul Cubukcu, University of Pennsylvania (United States)

8 Origami: Where Art, Devices, and Structures Merge

Richard Vaia, Air Force Research Laboratory (United States)

9 Micro- and Nano-Sensors and Materials for Oil and Gas Applications Joyce Wong, California Institute of Technology (United States)

10 Novel Harsh Environment Sensors for Energy Applications: Joint Session with Conferences 9467 and 9491

Michael P. Buric, National Energy Technology Laboratory

(United States) 
11 MAST: Bio-inspired Control: Joint Session with Conferences 9467, 9468, 9479

Christopher M. Kroninger, U.S. Army Research Laboratory

(United States)

William D. Nothwang, U.S. Army Research Laboratory (United States)

12 MAST: Scale Legged Locomotion: Joint Session with Conferences

9467, 9468, 9479

Christopher M. Kroninger, U.S. Army Research Laboratory

(United States)

William D. Nothwang, U.S. Army Research Laboratory (United States)

13 Novel Beam Control Applications and Techniques

Christopher C. Wilcox, U.S. Naval Research Laboratory (United States)

Matthew E. L. Jungwirth, Honeywell Automation \& Control Solutions

(United States)

14 Photonics Research at SPAWAR

David T. Wayne, Space and Naval Warfare Systems Center Pacific

(United States)

15 Keynote Session

Michael K. Rafailov, The Reger Group (United States)

16 Ultra-fast Bandgap Photonics

Michael K. Rafailov, The Reger Group (United States)

$17 \mathrm{THz}$ Photonics

Michael K. Rafailov, The Reger Group (United States)

18 Mid-IR Laser Photonics

Michael K. Rafailov, The Reger Group (United States)

19 Quantum Cascade Lasers and Spectroscopy

Michael K. Rafailov, The Reger Group (United States)

20 Laser Chemical Detection: Joint Session with Conferences 9467, 9455, 9486

Michael K. Rafailov, The Reger Group (United States)

21 Quantum Cascade Lasers: Joint Session with Conferences 9467, 9455, 9486

Michael K. Rafailov, The Reger Group (United States) 
Proc. of SPIE Vol. 9467 946701-16

Downloaded From: https://www.spiedigitallibrary.org/conference-proceedings-of-spie on 26 Apr 2023 Terms of Use: https://www.spiedigitallibrary.org/terms-of-use 


\section{Introduction}

The 2015 Micro- and Nanotechnology (MNT) Sensors, Systems, and Applications VII conference was, once again, fortunate to have an exciting program that spanned all 5 days (Monday through Friday) of the SPIE Defense and Security Symposium, held in Baltimore, Maryland, 20-24 April 2015.

True to its established tradition, a total of 21 Conference Sessions were successfully concluded within this conference on a broad spectrum of exciting topics ranging from: future trends in flexible, stretchable and transient electronics; bio-modulation achieved via low-intensity ultrasound and laser energy delivery; next generation graphene, transition metal chalcogenide and black phosphorous 2D materials and devices; surface-enhanced spectroscopies for ultrasensitive sensing; MNT-sensors and materials for oil and gas applications; harsh environment sensors for energy applications; micro-autonomous systems technology; novel beam control optics; ultra-fast bandgap photonics; terahertz and mid-IR photonics; quantum cascade lasers (QCLS) and their application to standoff chemical detection. Of particular interest was a new session on the emerging field of MNT Origami: fusing art and science to create novel devices and structures.

Successful joint sessions were conducted with the Sensors for Extreme Harsh Environments conference (9491), the Unmanned Systems Technology conference (9468), the Open Architecture/Open Business Model Net-Centric Systems and Defense Transformation conference (9479), the Chemical, Biological, Radiological, Nuclear, and Explosives (CBRNE) Sensing conference (9455), and the Advanced Environmental, Chemical, and Biological Sensing Technologies conference (9486).

It is our sincere hope that the papers within this proceedings volume will provide you, our reader, not only with a snapshot of the programmatic vision behind investments made in each MNT topic area, but also its current state of scientific and technological development. Enjoy!

Thomas George Saif Islam Achyut Dutta 
Proc. of SPIE Vol. $9467946701-18$

Downloaded From: https://www.spiedigitallibrary.org/conference-proceedings-of-spie on 26 Apr 2023 Terms of Use: https://www.spiedigitallibrary.org/terms-of-use 